\title{
Usefulness of rat-derived antigen in the serodiagnosis of Pneumocystis carinii infection
}

\author{
JEAN M. W. ChATTERTON, A. W. L. JOSS, T. H. PENNINGTON* and D. O. HO-YEN \\ Microbiology Department, Raigmore Hospital NHS Trust, Inverness IV2 3UJ and *Department of Medical \\ Microbiology, University of Aberdeen, Foresterhill, Aberdeen AB9 2ZD
}

\begin{abstract}
Sera from patients with likely and possible Pneumocystis carinii pneumonia (PCP) on the basis of clinical information and laboratory investigations were tested by immunoblotting to assess the usefulness of trophozoites in the serodiagnosis of PCP. IgG antibodies to 50-60- $\mathrm{kDa}$ proteins were demonstrated with cyst antigen, but antibodies to additional proteins of $61,70,82,95,99$ and $116 \mathrm{kDa}$ were found with trophozoite antigen. These bands were not demonstrated with control sera. IgG antibody to the 116kDa protein was found in 18 (46\%) of 39 sera from patients with possible PCP compared with $5(17 \%)$ of 30 sera from patients with likely PCP. There was no other significant difference between the two patient groups in detection of these proteins. Sera with higher indirect immunofluorescence assay (IFA) IgG titres were more likely to be immunoblot positive. Only 4 of 16 patients with likely PCP were IgG negative in the IFA; three of these were IgG immunoblot positive. In 4 of 10 patients with likely PCP and 6 of 15 patients with possible PCP, demonstration of IgM or IgA, or both, by IFA or immunoblotting provided evidence suggestive of current infection. This study confirms the usefulness of rat-derived antigen, especially trophozoite antigen, in PCP serology. The IgG IFA remains the most useful test, but IgM and IgA testing and immunoblotting can support the diagnosis.
\end{abstract}

\section{Introduction}

Genetic and antigenic diversity is being recognised increasingly in Pneumocystis carinii [1]. Gene sequencing and monoclonal antibody studies, particularly of the major surface glycoprotein, have identified differences between rat- and human-derived $P$. carinii and between isolates from rats and human patients [2]. Recognition of these differences has made the use of rat antigen in human serology controversial and has overshadowed earlier studies which concluded that $P$. carinii from rats and man have shared as well as distinct antigenic determinants $[3,4]$. In this laboratory, the results of 10 years' experience with rat-derived $P$. carinii in an indirect immunofluorescence assay (IFA) for detection of $\operatorname{IgG}$ antibodies to $P$. carinii have consistently demonstrated that it can be useful [5-8] and the test is now offered as a Reference Service. Before rat-derived antigen is abandoned because of antigenic heterogeneity, it seems sensible to assess its diagnostic usefulness.

Received 1 Oct. 1998; accepted 3 Nov. 1998.

Corresponding author: Dr J. M. W. Chatterton.
Trophozoites rather than cysts are associated with active $P$. carinii infection [9] yet the potential value of trophozoites in serological diagnosis remains to be investigated. A previous study showed that the most useful serological marker of $P$. carinii pneumonia (PCP) in immunosuppressed rats was demonstration of antibodies to proteins of 81 and $63 \mathrm{kDa}$ present in trophozoite but not in cyst antigen preparations [10]. The present study assessed the usefulness of immunoblotting with cyst and trophozoite rat-derived antigens in the serological diagnosis of human PCP.

\section{Materials and methods}

\section{$P$. carinii antigens}

PCP was induced in male Sprague-Dawley rats weighing $150 \mathrm{~g}$ (Harlan Olac, Bicester) by twiceweekly subcutaneous injection of $25 \mathrm{mg}$ of hydrocortisone acetate (The Boots Company, Nottingham). Tetracycline $(1 \mathrm{mg} / \mathrm{ml})$ supplemented with penicillin $(1 \mathrm{mg} / \mathrm{ml})$ was added to the water supply provided for the rats to protect against bacterial infection. The rats were immunosuppressed for 6-8 weeks and developed auto-infection. They were killed by intraperitoneal 
injection of pentobarbitone sodium (Veterinary Drug Co., York). Giemsa, toluidine blue $\mathrm{O}$ and indirect immunofluorescence assay (IFA) with rat anti- $P$. carinii antiserum and fluorescein-conjugated anti-rat globulin (Dako, Denmark) were used to identify $P$. carinii cysts and trophozoites in lung imprints, washings and gradient fractions [10].

The preparation of trophozoite-rich and cyst-rich fractions has been described previously [10]. P. carinii was eluted from minced, heavily infected lung by repeated stirring for $30 \mathrm{~min}$ in sterile phosphatebuffered saline, $\mathrm{pH} 7.3$, containing mixed antibiotics (PBSM). Eluates containing the most parasites and the least host cell contamination were washed three times in PBSM by centrifugation at $2000 \mathrm{~g}$ for $15 \mathrm{~min}$ and resuspended in PBSM containing $1 \mathrm{mM}$ ethylene diamine tetra-acetic acid (EDTA). The suspensions were forced three times through a fine bore needle and gross particulate contamination was removed before digestion with collagenase (Type A; Sigma) $1.5 \mathrm{mg} / \mathrm{ml}$ at $37^{\circ} \mathrm{C}$ for $1.5 \mathrm{~h}$ with gentle agitation. Discontinuous gradients of three 5-ml layers of Percoll (Pharmacia) in PBSM containing $1 \mathrm{mM}$ EDTA (density $36 \%, 16 \%$ and $8 \%$, respectively) were loaded with $5 \mathrm{ml}$ of sample and centrifuged at $2000 \mathrm{~g}$ for $15 \mathrm{~min}$. The interphases and intervening Percoll layers were collected individually from the top of the gradients by angled pipette, washed three times in PBSM and the relative numbers of cysts and trophozoites were counted. Fractions were classified according to the relative numbers of cysts and trophozoites and stored at $-20^{\circ} \mathrm{C}$. Fractions containing $>90 \%$ trophozoites and $>80 \%$ cysts were used in this study. Like fractions from the processed tissue of several animals were pooled to prepare sufficient antigen to test panels of sera. Fractions were pooled only when they had been examined individually by SDS-PAGE and immunoblotting with rat anti- $P$. carinii positive control serum and had given characteristic banding patterns as described previously [10]. The antigen was distributed in aliquots and stored at $-20^{\circ} \mathrm{C}$ until used for immunoblotting.

\section{Sera}

Patient specimens were submitted for investigation of P. carinii infection by hospitals in Scotland, England and Ireland. Samples were selected for immunoblotting on the basis of the patients' clinical history and their IFA results. Sixty-nine sera from 50 patients were tested for IgG antibodies in two groups. (i) A panel of 30 sera from 16 patients with likely PCP; ten patients had clinical PCP and $P$. carinii was demonstrated in respiratory samples in this or the submitting laboratory; six patients had good clinical evidence of PCP and a good response to treatment. (ii) A panel of 39 sera from 34 patients with possible PCP; the patients had limited clinical evidence to support a diagnosis of PCP and were negative or not investigated for the presence of $P$ carinii in respiratory samples. The patients suffered from various conditions, but were predominantly those with malignancies and transplants and did not include HIV/AIDS patients. A control panel of 48 sera from healthy blood donors and 18 sera from blood samples collected for therapeutic purposes was also tested.

Thirty-two sera from 25 of the 50 patients were tested for IgA and IgM antibodies: (i) 15 sera from 10 of the patients with likely PCP; (ii) 17 sera from 15 of the patients with possible PCP. A control panel of 10 blood donor sera was also tested.

\section{Immunofluorescence assay}

Serum antibodies were measured by an indirect immunofluorescence technique as reported previously [7]. The antigen was prepared from unfractionated $P$. carinii washings and contained cysts and trophozoites. Sera were tested at dilutions ranging in two-fold steps from 1 in 8 to 1 in 256 for IgG antibodies and from 1 in 8 to 1 in 32 for IgM and IgA antibodies with fluorescein-labelled sheep anti-human IgG, IgM and IgA (Scottish Antibody Production Unit, Carluke).

\section{SDS-PAGE and immunoblotting}

Antigens were boiled for $5 \mathrm{~min}$ in a lysis buffer containing SDS $2 \%, 0.06 \mathrm{M}$ Tris-HC1 (pH 6.8), 2mercapto-ethanol $5 \%$, glycerol $10 \%$ and bromophenol blue $0.001 \%$. Electrophoresis was performed at a constant current of $6 \mathrm{~mA}$ for $16 \mathrm{~h}$ with a stacking $3.5 \%$ gel and a separating $12 \%$ slab gel in a discontinuous SDS buffer system. A mol. wt standard mixture containing myosin, $\beta$-galactosidase, phosphorylase $\mathrm{B}$, bovine albumin, egg albumin and carbonic anhydrase (SDS6H; Sigma) was run on each gel. Separated proteins were transferred to nitrocellulose sheets (pore size $0.45 \mu \mathrm{m}$ ) in an LKB 2005 electroblotting unit at $4^{\circ} \mathrm{C}$ for $2 \mathrm{~h}$ at $0.8 \mathrm{~A}$. Lanes containing the mol. wt standards were stained with amido black (Sigma) and used as standards to determine the mol. wts of test proteins. The remainder of the sheet was blocked in PBS containing non-fat milk $5 \%$ for $1 \mathrm{~h}$, rinsed in PBS containing Tween $200.05 \%$ (PBST), dried and stored in the dark at room temperature. Strips cut from the sheet were incubated for $16 \mathrm{~h}$ at room temperature with a 1 in 100 or 1 in 500 dilution of serum for IgG and 1 in 50 dilution of serum for IgM and IgA in PBST containing non-fat milk 5\% (PBSN). Strips were washed five times in PBST, then incubated for $2 \mathrm{~h}$ at room temperature in a 1 in 1000 dilution of goat anti-human IgG, IgM or IgA peroxidase conjugate (Sigma) in PBSN. After five washes in PBST and two in PBS, strips were immersed in substrate (4-chloro-1napthol $0.006 \%$ in methanol, $\mathrm{H}_{2} \mathrm{O}_{2} \quad 0.014 \%$ in PBS) for $10 \mathrm{~min}$, washed twice in PBST, once in distilled water, left for $30 \mathrm{~min}$ in distilled water and dried. 


\section{Statistical analysis}

Where appropriate, data were subjected to statistical analysis by $\chi^{2}$ with Yates' correction for small samples.

\section{Results}

\section{IgG immunoblotting}

When 17 blood donor and 14 patient sera were tested at a 1 in 100 dilution, cyst-rich antigen produced weak bands in the 50-60-kDa range whereas trophozoite-rich antigen produced a range of bands. As trophozoite antigen was the more reactive, it was used for immunoblotting and cyst antigen was not pursued. With blood donor sera tested at a 1 in 100 dilution, a range of bands was demonstrated. A broad band between 50 and $60 \mathrm{kDa}$ was dominant and present in 24 of 48 sera. Just 6 of 48 blood donor sera were immunoblot-negative; only 3 of 28 of these sera showed specific IgG antibody to $P$. carinii by IFA and only at a titre of 1 in 8 . Eighteen sera from blood samples taken for therapeutic purposes showed a similar pattern of reactivity. When four of these control sera were tested at dilutions of 1 in 100,1 in 200,1 in 400 and 1 in 500 , bands $>70 \mathrm{kDa}$ were virtually eliminated and the intensity of bands at $50-60 \mathrm{kDa}$ was reduced. This pattern was confirmed by repeat testing of 16 selected blood donor sera which were reactive at dilutions of 1 in 100 and 1 in 500 (Table 1). All sera were subsequently tested at a 1 in 500 dilution to eliminate background antibody.

The major IgG bands detected in trophozoite-rich $P$. carinii antigen with patient sera are shown in Table 1 and Figs. 1 and 2. Between one and eight prominent IgG immunoblotting bands were demonstrated. The intensity of the bands was variable and patterns were often weaker in patients with likely PCP than in patients with possible PCP (Figs. 1 and 2). There was no significant difference in the number of bands demonstrated by sera in the two patient groups $\left(\chi^{2}, \mathrm{p}>0.5\right)$. Antibody to the $82-\mathrm{kDa}$ protein was more common in patients with likely PCP. The band was demonstrated in $15(50 \%)$ of 30 sera from patients with likely PCP compared with $15(38 \%)$ of 39 sera from patients with possible PCP. Although this difference was not significant $\left(\chi^{2}, p>0.1\right)$, this may be because of the small number of patients. There was also no significant difference in the frequency of detection of the $50-60-, 61-, 70-, 95-$ or $99-\mathrm{kDa}$ bands in patients with likely and possible PCP $\left(\chi^{2}, \mathrm{p}>0.5\right)$. The $116-\mathrm{kDa}$ band was identified more frequently $\left(\chi^{2}, p<0.02\right)$ in sera from patients with possible PCP.

Patients' sera had IgG IFA titres from $<8$ to 256 . Eighteen of 30 sera from patients with likely PCP were seropositive $(\geqslant 16)$ by IgG IFA and three patients seroconverted. Thirty-four of 39 sera from patients with possible PCP were seropositive by IgG IFA. There was no significant difference in IgG IFA titres between patients with likely and possible PCP $\left(\chi^{2}, p>0.5\right)$. Sera with higher IFA titres were more likely to be immunoblot positive $\left(\chi^{2}, \mathrm{p}<0.02\right) ; 14$ $(56 \%)$ of 25 sera with IFA titres $\leqslant 16$ were immunoblot positive compared with $11(65 \%)$ of 17 with IFA titres of 32 and 26 (96\%) of 27 with higher titres.

The IgG IFA titres and immunoblotting patterns in sera from individual patients with likely PCP are shown in Table 2. Correlation of IgG IFA titres and IgG bands was variable. Patients 11,15 and 16 were IFA IgG antibody negative, but produced positive blots recognising up to six bands. In two of these patients (nos. 15 and 16) $P$. carinii was demonstrated in broncho-alveolar lavage by PCR, although IFA gave equivocal results.

\section{$\operatorname{Ig} M$ and $\operatorname{Ig} A$}

The 10 blood donor sera were IgA and IgM negative by IFA and immunoblotting. Patients' sera had IgA and IgM titres of $<8-32$ in the IFA. Sera demonstrating IgM or IgA immunoblotting bands, or both, recognised the same range of proteins as $\mathrm{IgG}$, but the patterns were weaker. In four of 10 patients with likely PCP, two sera demonstrated IgM and IgA, three IgM only and one IgA only. In six of 15 patients with possible PCP, two sera demonstrated both IgM and IgA, six IgM only and none IgA only. Sera with IFA titres of 8 produced between two and six bands on blotting. All the sera that demonstrated IgA and IgM bands on blotting had IgG, IgM and IgA IFA titres $\geqslant 8$.

\section{Discussion}

The need for a serological test for PCP has long been acknowledged $[2,4,11,12]$ and has been emphasised

Table 1. IgG bands detected in $P$. carinii trophozoite antigen with blood donor and patient sera

\begin{tabular}{lccccccccccc}
\hline & Number of & \multicolumn{8}{c}{ Number (\%) of sera with antibody to proteins (kDa) } \\
\cline { 3 - 12 } Subjects (n) & sera & 116 & 99 & 95 & 82 & 70 & 61 & $50-60$ & 40 & None \\
\hline Blood donors (16) & 16 & 0 & 0 & 0 & 0 & 0 & 0 & $10(62)$ & $2(12)$ & $6(4)$ \\
Likely PCP (16) & 30 & $5(17)$ & $10(33)$ & $9(30)$ & $15(50)$ & $7(23)$ & $7(23)$ & $17(57)$ & 0 & $8(27)$ \\
Possible PCP (34) & 39 & $18(46)$ & $12(31)$ & $12(31)$ & $15(38)$ & $9(23)$ & $11(28)$ & $26(66)$ & $2(5)$ & $10(26)$ \\
Total (66) & 85 & $23(27)$ & $22(26)$ & $21(25)$ & $30(35)$ & $16(19)$ & $18(21)$ & $53(62)$ & $4(5)$ & $24(28)$ \\
\hline
\end{tabular}




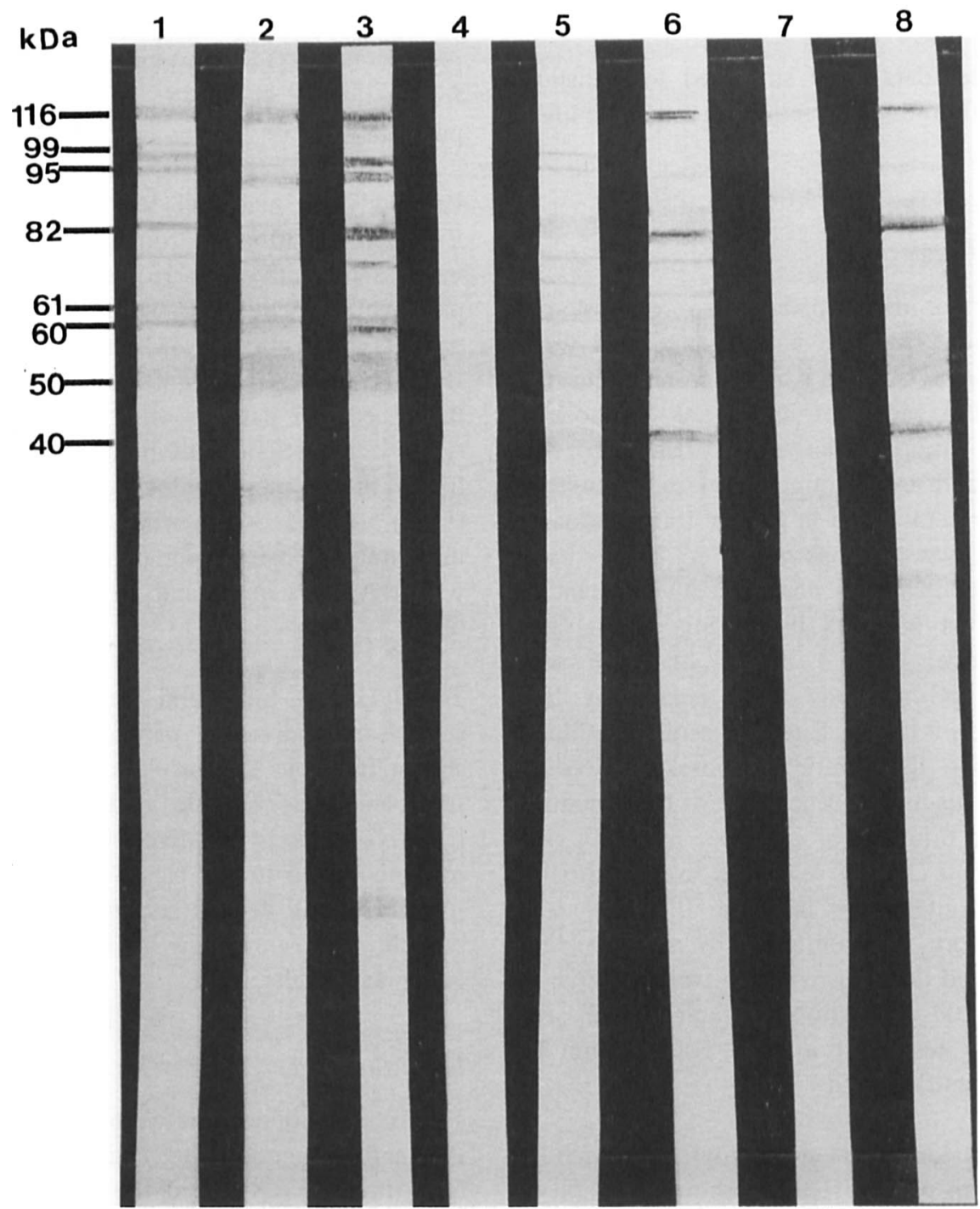

Fig. 1. IgG Western immunoblot of $P$. carinii trophozoite-rich antigen with sera from patients with possible PCP. Lanes $1,2,3,5$, single sera; 4 and 7,6 and 8 , paired sera.

by studies showing that the presence of $P$. carinii in respiratory samples does not always equate with significant clinical disease [13-15]. In the past there has been little agreement on the best source and method of antigen preparation [3] and this debate continues. Human-derived $P$. carinii would be the preferred source of antigen for serological studies [4, 12], but limitations of culture, supply and control still restrict its use. Rat-derived $P$. carinii is not only available, but this laboratory and others have shown that it can be used in an IFA to detect specific $P$. carinii antibodies in human sera. However, the IFA does not allow discrimination of specific antibodies to cysts or trophozoites, and common antigens could easily mask unique antigens. This laboratory has some evidence that antibody to trophozoites may be more important than antibody to cysts $[3,10]$. By looking at cysts and trophozoites separately by immunoblotting, earlier studies confirmed that they have different profiles [10] and demonstrated the importance of trophozoite antigen in $P$. carinii serology, as cyst antigen was poorly reactive while trophozoite antigen produced a range of bands with patients' sera.

The IgG immunoblotting patterns of sera from patients with likely and possible PCP were similar, but significantly different from blood donor sera. Antibody to $50-60-, 61-, 70-, 82-, 95-, 99-$ and $116-\mathrm{kDa}$ proteins was present in patients' sera whereas only the $50-60-\mathrm{kDa}$ protein was demonstrated in control sera. This accords well with the antibody to 50-60-, 63-, $81-, 104-$ and $124-\mathrm{kDa}$ proteins found in sera from immunosuppressed rats with PCP [10]. In patients' sera, the $61-\mathrm{kDa}$ protein, (probably equivalent to the 


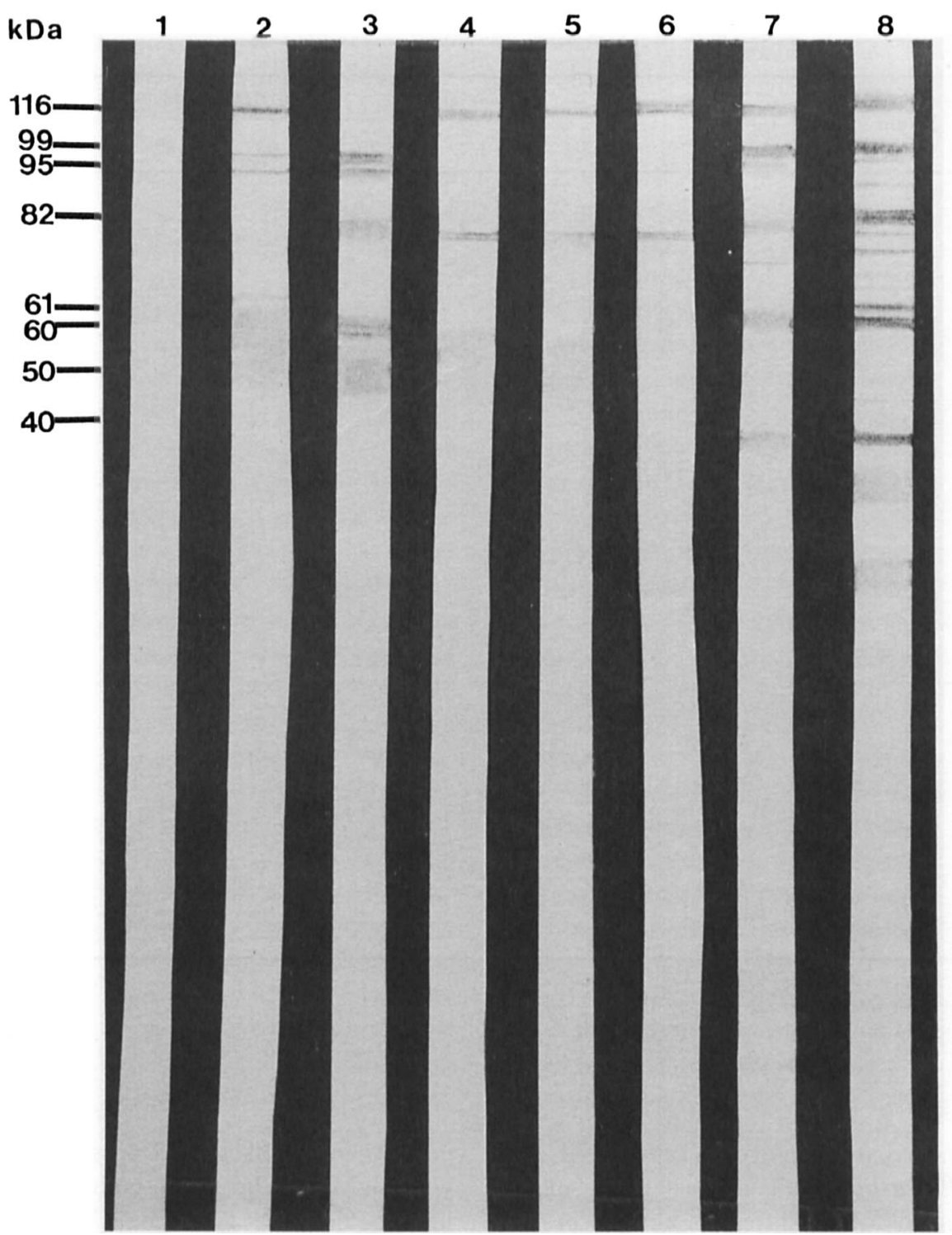

Fig. 2. IgG Western immunoblot of $P$. carinii trophozoite-rich antigen. Lanes 1-3, 8, sera from patients with possible PCP; 4-6, sera from a patient with likely PCP; 7, serum from a patient with likely PCP.

rat $63-\mathrm{kDa}$ protein) was equally recognised by both patient groups, but antibody to the $82-\mathrm{kDa}$ protein, (equivalent to the rat $81-\mathrm{kDa}$ protein) was most prominent in patients with likely PCP; this may be a useful marker of current infection. Like other studies [12], this study found variability in blotting patterns, and sera from patients with likely PCP sometimes produced weaker and fewer bands than sera from patients with possible PCP. By testing sera at a high dilution to eliminate background antibody, detection of low levels of antibody in these immunosuppressed patients may have been compromised.

The $116-\mathrm{kDa}$ protein probably represents the major surface glycoprotein (MSG) of rat-derived $P$. carinii $[2,4]$. This protein is by far the best studied and has been cloned and sequenced $[1,2]$. It has been suggested that the equivalent human-derived $P$. carinii protein would be useful in serology $[2,4]$, but this laboratory found that antibody to rat MSG was of limited use, as it was more prominent in patients with possible than likely $P$. carinii pneumonia. This is perhaps not surprising as trophozoite antigen was found to be more useful than cyst antigen and MSG is less abundant in trophozoites than cysts [16]. MSG gave variable results when used to characterise different human isolates with monoclonal antibodies, which may be useful in distinguishing isolates, but is a disadvantage in epidemiological studies [2]. In a major immunoblotting study Peglow et al. identified major immunoblotting bands of 40,66, 92 and $116 \mathrm{kDa}$ in human $P$. carinii; again, it was not the $116-\mathrm{kDa}$ MSG which proved to be the major marker of $P$. carinii, but rather a $40-\mathrm{kDa}$ protein. This study 
Table 2. IgG IFA titres and immunoblotting bands detected with $P$. carinii trophozoite-rich antigen and sera from 16 patients with likely $P$. carinii infection

\begin{tabular}{|c|c|c|c|c|c|c|c|c|c|c|}
\hline \multirow{2}{*}{$\begin{array}{l}\text { Patient } \\
\text { no. }\end{array}$} & \multirow{2}{*}{$\begin{array}{c}\text { Serum } \\
\text { no. }\end{array}$} & \multirow{2}{*}{$\begin{array}{l}\text { Times } \\
\text { (days) }\end{array}$} & \multirow{2}{*}{$\begin{array}{l}\text { IFA } \\
\text { IgG }\end{array}$} & \multicolumn{7}{|c|}{ Bands detected $(\mathrm{kDa})$} \\
\hline & & & & 116 & 99 & 95 & 82 & 70 & 61 & $60-50$ \\
\hline \multirow[t]{3}{*}{$1^{*}$} & 1 & 0 & 64 & - & - & - & + & + & - & + \\
\hline & 2 & 9 & 128 & ++ & + & + & + & + & + & + \\
\hline & 3 & 35 & 64 & + & + & - & $t+$ & + & + & + \\
\hline \multirow[t]{3}{*}{$2^{*}$} & 1 & 0 & $<8$ & - & - & - & - & - & - & - \\
\hline & 2 & 11 & 16 & - & - & - & - & - & - & - \\
\hline & 3 & 13 & 8 & - & - & - & - & - & - & - \\
\hline \multirow[t]{2}{*}{$3^{*}$} & 1 & 0 & 64 & - & - & - & + & + & - & + \\
\hline & 2 & 3 & 64 & - & - & - & + & - & - & + \\
\hline \multirow[t]{4}{*}{$4^{*}$} & 1 & 0 & 32 & - & - & - & - & - & - & + \\
\hline & 2 & 3 & 32 & + & $+1-$ & $+1-$ & - & - & - & + \\
\hline & 3 & 14 & 16 & - & - & - & $+1-$ & - & - & - \\
\hline & 4 & 16 & 16 & - & - & - & $+1-$ & - & - & - \\
\hline $5^{*}$ & 1 & - & $<8$ & - & - & - & - & - & - & - \\
\hline $6^{*}$ & 1 & - & 16 & - & - & - & - & - & - & + \\
\hline $7^{*}$ & 1 & - & 128 & - & - & - & - & - & - & + \\
\hline $8^{*}$ & 1 & - & 32 & - & $+1-$ & $+1-$ & $+1-$ & $+1-$ & $+1-$ & $+1-$ \\
\hline $9^{*}$ & 1 & - & 128 & - & - & - & + & - & - & + \\
\hline $10^{*}$ & 1 & - & 32 & - & - & - & - & - & - & - \\
\hline \multirow[t]{3}{*}{11} & 1 & 0 & $<8$ & - & $+1-$ & $+1-$ & $+1-$ & - & - & - \\
\hline & 2 & 9 & $<8$ & - & $+1-$ & $+1-$ & $+1-$ & - & - & - \\
\hline & 3 & 10 & $<8$ & - & $+1-$ & $+1-$ & $+1-$ & - & + & - \\
\hline \multirow[t]{2}{*}{12} & 1 & 0 & $<8$ & - & - & - & - & - & - & - \\
\hline & 2 & 35 & 128 & - & - & - & - & - & - & + \\
\hline \multirow[t]{2}{*}{13} & 1 & 0 & $<8$ & - & - & - & - & - & - & - \\
\hline & 2 & 47 & 32 & - & - & - & - & - & - & + \\
\hline \multirow[t]{2}{*}{14} & 1 & 0 & $<8$ & - & - & - & - & - & - & - \\
\hline & 2 & 10 & 128 & + & - & + & - & + & - & + \\
\hline \multirow[t]{2}{*}{$15 \dagger$} & 1 & 0 & $<8$ & - & + & - & + & - & $+1-$ & + \\
\hline & 2 & 52 & $<8$ & + & + & + & + & + & + & + \\
\hline $16 \dagger$ & 1 & - & $<8$ & - & $+1-$ & $+1-$ & $+1-$ & $+1-$ & $+1-$ & $+1-$ \\
\hline
\end{tabular}

Intensity of bands: ++ very strong; + strong; $+/$ - faint; - absent.

$* P$. carinii demonstrated in respiratory samples by IFA.

$\dagger P$. carinii demonstrated in broncho-alveolar lavage by PCR.

also confirmed the cross-reactivity of rat-derived and human-derived $P$. carinii antigens, as these major bands were detected when different human or rat isolates were tested, and when different serum specimens from human patients and rats were used as source antibody [12]. It is possible that the $82 \mathrm{kDa}$ rat-derived $P$. carinii protein prominent in the present study is a variant of this $40-\mathrm{kDa}$ human-derived $P$. carinii protein.

There is little published information on IgA testing for PCP [17]. Active IgM responses to a $40-\mathrm{kDa}$ antigen have been detected in PCP patients [12], but this laboratory [7] and others [18, 19] have previously failed to detect IgM by IFA. The highest IgM titre found in this study was 32, and in $9(75 \%)$ of 12 IgM-positive sera titres were only 8 and must be considered doubtful positive results. Similarly in 11 $(69 \%)$ of 16 IgA-positive sera, titres were only 8 . Immunoblotting did not produce more IgM- or IgApositive results. Positive immunoblots supported the specificity of any IFA titres, even as low as 8 . Demonstration of IgM and IgA suggests primary or reactivated infection, and the low titres favour a diagnosis of reactivation. However, the results would also be consistent with re-infection $[1,12,14]$. There was no significant difference in the proportion of patients in the likely and possible PCP patient groups with $\operatorname{IgM}$ or $\operatorname{IgA}$, or both, detected by IFA or immunoblotting. This could indicate that there is some imprecision in distinguishing the two patient groups and that six of the 15 possible PCP cases merit re-classification to the likely group on the basis of their IgA and IgM results.

Seroconversion or a significant rise in IgG antibody provides the best evidence of current infection [9, 20] and in two of the three patients who demonstrated this response by IFA, immunoblotting with trophozoite antigen was negative. However, in three of the four patients with likely PCP who were seronegative by IFA, IgG antibody was detected by immunoblotting. This may reflect the stage or severity of the infection. In two of the patients that gave positive immunoblots with no detectable IgG IFA antibody, respiratory secretions were negative for $P$. carinii when examined by an IFA to detect cysts, but positive by PCR which detects cysts and trophozoites [21]. It is likely that the antibody response is to trophozoite antigens early in infection, and then broadens to include antibodies to antigens common to cysts and trophozoites. Thus, immunoblotting with trophozoite antigen may be more useful early in infection when trophozoites predominate and antibody levels are low, and IFA may be 
more appropriate later in infection to detect antibody to both cysts and trophozoites. The results described in this paper confirm both the usefulness of ratderived antigen in serological diagnosis of PCP [5-8] and the importance of trophozoite antigens in the future development of PCP serology.

We thank R. Evans, D. Ashburn and Lesley Dargie for their assistance and helpful co-operation; Debbie Gilham and Kim Cahill for invaluable secretarial assistance; A. McGinley for producing the figures; and the Scottish Home and Health Department (SHHD grant no. $\mathrm{K} / \mathrm{MRS} / 50 / \mathrm{C} 1828$ ) for financial support. We are grateful to the many clinicians and laboratories throughout Britain who have referred specimens to us.

\section{References}

1. Stringer JR, Walzer PD. Molecular biology and epidemiology of Pneumocystis carinii infection in AIDS. AIDS 1996; 10: 561-71

2. Smulian AG, Keely SP, Sunkin SM, Stringer JR. Genetic and antigenic variation in Pneumocystis carinii organisms: tools for examining the epidemiology and pathogenesis of infection. $J$ Lab Clin Med 1997; 130: 461-8.

3. Chatterton JMW, Joss AWL, Davidson MM, Ho-Yen DO. Why have Pneumocystis carinii trophozoites been ignored? $J$ Clin Pathol 1990; 43: 265-8.

4. Lundgren B. Pneumocystis carinii: antigenic, immunological, and molecular characterization. Dan Med Bull 1994; 41: 306-18.

5. Williamson JMW. The diagnosis of Pneumocystis carinii infections. Commun Dis Scot Wkly Rep 1983; 20: VII.

6. Chatterton JMW, Joss AWL, Ho-Yen DO. Serological results in 24 cases of proven or likely Pneumocystis carinii pneumonia. Commun Dis Scot Wkly Rep 1988; 44: 5-7.

7. Chatterton JMW, Joss AWL, Williams H, Ho-Yen DO. Pneumocystis carinii antibody testing. J Clin Pathol 1989; 42: $865-8$.

8. Evans R, Dargie L, Chatterton JMW, Ho-Yen DO. Serological diagnosis of $P$. carinii infection. Commun Dis Environ Hlth Scot Wkly Rep 1993; 27: 5-8.
9. Ho-Yen DO, Chatterton JMW, Joss AWL. Laboratory diagnosis of Pneumocystis carinii pneumonia. J Med Microbiol 1995; 42: $231-2$.

10. Chatterton JMW, Joss AWL, Pennington TH, Ho-Yen DO. Differences exist in the immunoblotting profiles of cyst and trophozoite antigens of Pneumocystis carinii. J Med Microbiol 1995; 42: 120-6.

11. Jameson B. Serology of Pneumocystis carinii. In: Young LS (ed) Pneumocystis carinii pneumonia: Pathogenesis, diagnosis, treatment. Lung biology in health and disease, vol 22. New York, Marcel Dekker. 1984: 97-106.

12. Peglow SL, Smulian AG, Linke MJ et al. Serologic responses to Pneumocystis carinii antigens in health and disease. J Infect Dis 1990; 161: 296-306.

13. Contini C, Romani R, Vullo V, Delia S, Sorice F. Does laboratory recovery of Pneumocystis carinii always mean clinically significant disease? $A I D S$ 1992; 6: 1558-9.

14. Matusiewicz SP, Fergusson RJ, Greening AP, Crompton GK, Burns SM. Pneumocystis carinii in bronchoalveolar lavage fluid and bronchial washings. $B M J 1994 ; 308$ : 1206-7.

15. Weig M, Klinker $\mathrm{H}$, Bögner $\mathrm{BH}$, Meier A, Gross U. Usefulness of PCR for diagnosis of Pneumocystis carinii pneumonia in different patient groups. $J$ Clin Microbiol 1997; 35: 1445-9.

16. Pascale JM, Shaw MM, Bartlett MS, Smith JW, McLaughlin GL. Western blot analysis of rat Pneumocystis carinii antigens using convalescent rat sera and rabbit anti-gp120. J Eukaryot Microbiol 1996; 43: 30S.

17. Nielsen PB, Mojon M. Enzyme linked immunosorbent assay compared with indirect immunofluorescence test for detection of Pneumocystis carinii specific immunoglobins G, M and A APMIS 1988; 96: 649-54.

18. Shepherd V, Jameson B, Knowles GK. Pneumocystis carinii pneumonitis: a serological study. J Clin Pathol 1979; 32: 773-7.

19. Tanabe $\mathrm{K}$, Furuta $\mathrm{T}$, Ueda $\mathrm{K}$, Tanaka $\mathrm{H}$, Shimada $\mathrm{K}$. Serological observations of Pneumocystis carinii infection in humans. J Clin Microbiol 1985; 22: 1058-60.

20. Elvin K, Björkman A, Heurlin N, Eriksson B-M, Barkholt L, Linder E. Seroreactivity to Pneumocystis carinii in patients with AIDS versus other immunosuppressed patients. Scand $J$ Infect Dis 1994; 26: 33-40.

21. Evans R, Joss AWL, Parratt D, Pennington TH, Ho-Yen DO. The role of a nested polymerase chain reaction in the diagnosis of Pneumocystis carinii pneumonia. J Clin Pathol: Mol Pathol 1995; 48: M347-M350. 